\title{
Mujer y liderazgo en universidades privadas del estado de Hidalgo, México
}

\section{Women and leadership in private universities in the state of Hidalgo, Mexico}

Tirso Javier Hernández Gracia; Danae Duana Avila²;

Enrique Martínez Muñoz ${ }^{3}$; Bernardino Martínez Muñoz ${ }^{4}$

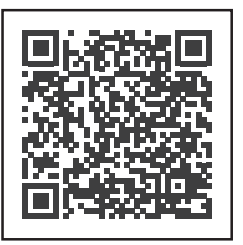

\section{Palabras clave:}

Mujer, liderazgo, oportunidades, alicientes, desigualdades, experiencias en el puesto, universidades privadas.

Articulo de investigación:

Fecha de recepción: 06/05/2020

Fecha de aceptación: 01/06/2020

Esta publicación se encuentra bajo licencia:

Creative Commons ReconocimientoNoComercialSinObraDerivada 4.0 Internacional

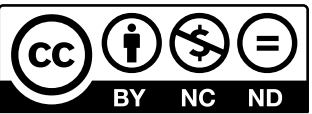

\section{Resumen}

Los puestos de dirección en cualquier empresa, sea pública o privada, generalmente son ocupados por hombres, resultando complicado para una mujer el acceso a esos cargos. En México, este tipo de situaciones aún se encuentran presentes, ya que por un lado, el personal femenino enfrenta problemas asociados a la falta de oportunidades de asumir cargos de jefaturas, y por otro, cuando finalmente lo logran, a veces existen desigualdades entre ambos géneros. La investigación tiene como propósito identificar cuáles son los factores más relevantes que se encuentran relacionados con la poca participación de este género en los mandos directivos, así como los principales alicientes y desigualdades que han experimentado. La muestra por conveniencia fue de cincuenta lideresas que ostentan cargos importantes en veinte universidades privadas mexicanas del estado de Hidalgo. El estudio es cuantitativo, descriptivo y transaccional y se realizó durante el

Doctor en Ciencias Administrativas, Universidad Autónoma del Estado de Hidalgo, México, thernan@uaeh.edu.mx, https://orcid.org/0000-0003-0425-0800

2 Doctor en Ciencias Económicas, Universidad Autónoma del Estado de Hidalgo, México, danae@uaeh.edu.mx, https://orcid.org/0000-0003-2286-2843

3 Doctor en Ciencias Administrativas, Universidad Autónoma del Estado de Hidalgo, México, enriquemarvar@gmail.com, https://orcid.org/0000-00016418-5292

4 Maestro en Administración, Universidad Autónoma del Estado de Hidalgo, México, bernardino_martinez@uaeh.edu.mx, https://orcid.org/0000-00027296-1666 


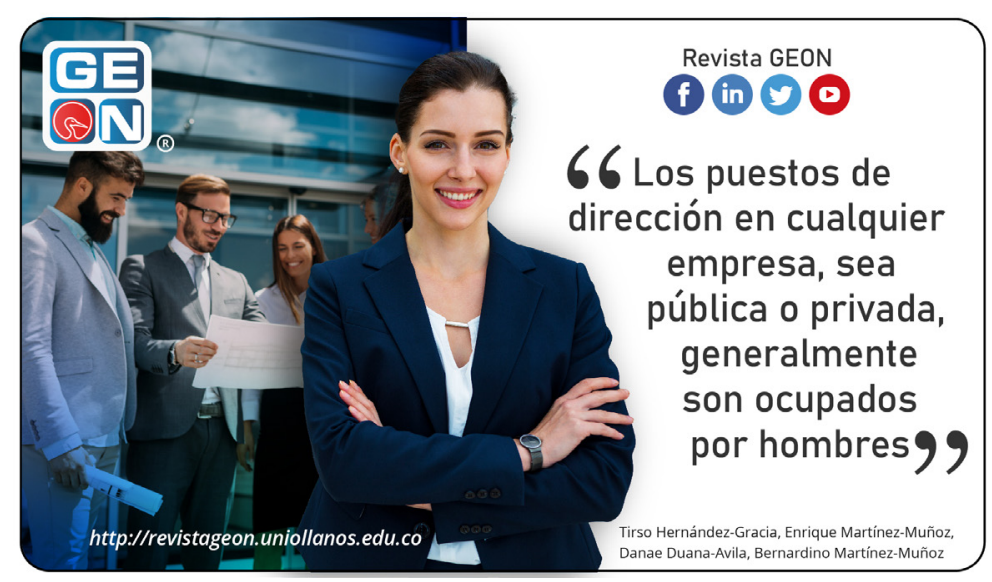

año 2018-2019. Se aplicó un cuestionario basado en el desarrollado por Bibiana M. Orjuela y David Z. Goyeneche en el 2014.Los resultados indican que aún es frecuente observar que las oportunidades de ocupar un nivel directivo es más favorable para los hombres, por razones que tienen que ver con los cuidados maternos. Los alicientes y desigualdades que más han enfrentado son la ayuda económica al núcleo familiar y la inequidad en los sueldos. Se concluye que debe concientizarse más a los dueños de las empresas para ser más equitativos en las oportunidades y condiciones dentro de un cargo de alto nivel.

Palabras claves: Mujer, liderazgo, oportunidades, alicientes, desigualdades, experiencias en el puesto, universidades privadas.

\section{Abstract}

Management positions in any company, whether public or private, are generally held by men, making it difficult for a woman to access these positions. In Mexico, these types of situations are still present, since on the one hand, female personnel face problems associated with the lack of opportunities to assume leadership positions, and on the other, when they finally succeed, and there are sometimes inequalities between the two genders. The purpose of the research is to identify which are the most relevant factors that are related to the low participation of this gender in management, as well as the 
Cómo citar este artículo /

Toreference this article:

Hernández-Gracia, T., Duana Avila, D., Martínez Muñoz, E., \& Martínez Muñoz, B. (2020). Mujer y liderazgo en universidades privadas del estado de Hidalgo, México. Revista GEON (Gestión, Organizaciones Y Negocios), 7(2), 1-12. https://doi. org/10.22579/23463910.213 main incentives and inequalities that they have experienced. The sample for convenience was 50 female leaders who hold important positions in three Mexican private universities in the state of Hidalgo. The study is quantitative, descriptive and transactional and was carried out during the 2018-2019 year. A questionnaire based on the one developed by Bibiana M. Orjuela and David Z. Goyeneche in 2014 was applied. The results indicate that it is still frequent to observe that the opportunities to occupy a managerial level are more favorable for men, for reasons that have to do with maternal care. The incentives and inequalities that they have faced the most are economic aid to the family nucleus and inequality in wages. It is concluded that business owners should be made more aware of themselves in order to be more equitable in opportunities and conditions within a high-level position.

Keywords: Women, leadership, opportunities, incentives, inequalities, job experiences, private universities.

\section{Introducción}

En México, al igual que en varios países de América Latina y del mundo, las mujeres enfrentan problemas para poder insertarse en el marcado laboral, toda vez que aún se mantiene arraigada la presencia de una identidad particularmente masculina (Buquet y Moreno, 2017). Esta situación se explica en parte por la masculinidad hegemónica, derivada de la teoría de la hegemonía cultural, donde se analiza las relaciones de poder por medio de los cuales un grupo social reclama y sostiene una posición de liderazgo dominante en una jerarquía social (Díez, 2015). La presencia de las mujeres en los puestos directivos ha mejorado con el paso del tiempo. No obstante, poco a poco la participación de las mujeres en puestos de responsabilidad va evolucionando, auqnue no es suficiente, a pesar de los efectos beneficiosos que la misma podría tener, aún hay diferenciasaún entre hombres y mujeres (Bonnafé, 2016; Biedma, 2017; Ocañas, 2019).

En relación con la Confederación de Cámaras Nacionales de Comercio, Servicios y Turismo (Concanaco Servytur México), Al primer trimestre de 2017 la Población Económicamente Activa (PEA) en el estado de Hidalgo fue de 456,744 mujeres, es decir, aproximadamente un $37 \%$ de la población total, quienes laboran en un 
contexto de pobreza y desigualdad. (Concanaco Servytur México, 2017). No se tienen datos precisos sobre cuantas de esas mujeres que laboran ocupan puestos directivos dentro de las empresas, no obstante, el Gobierno del estado de Hidalgo, México, durante la gestión del actual Gobernador, Lic. Omar Fayad Meneses, a capacitado a más de 73 mil mujeres en diversas áreas como la administración con el fin de fomentar la cultura de inclusión en el mercado laboral (Independiente, 2020).

El peso cultural que aún prevalece en diversas ciudades, pueblos, comunidades y barrios, sobre el rol que juega la mujer dentro de la sociedad y de manera específica en la familia, se convierten en barreras para que pueda tener acceso a un cargo importante dentro de la empresa (Labarcés Ballestas, 2015). También pueden estar presentes otros aspectos como el acoso, el estatus civil, la edad, entre otros, que se convierten en reglamentos o simplemente políticas que desfavorecen la participación del personal femenino en el mercado laboral. Algunos autores le llaman formas de discriminación laboral, las cuales se hacen patentes a través de procesos relacionados con los salarios, seguridad, prestaciones, entre otros (Horbath y Gracia, 2014).

Es por ello que resulta necesario el identificar, a través de la opinión de las lideresas que ocupan cargos directivos actualmente, su opinión sobre las oportunidades que hoy en día tienen las mujeres y sus experiencias, alicientes y desigualdades que han vi- vido durante el lapso de tiempo que han ocupado esos cargos de alto nivel, en este caso, en las universidades privadas del estado de Hidalgo, México.

\section{Contexto teórico}

La gestión estratégica de recursos humanos es un proceso complejo que está en constante evolución (O'riordan, 2017). Hoy en día, el mercado laboral requiere líderes vanguardistas que coordinen las actividades de sus subordinados, establezcan objetivos y metas alcanzables, así como, elegir parte del personal que van a laborar con ellos y se mantengan atentos a los cambios que imponga el entorno donde se desarrolla la empresa (Universia México, 2018).

Kemal (2015) comenta que los líderes más exitosos requieren otros rasgos, comportamientos y habilidades para el liderazgo, encausando sus esfuerzos hacia el control de las emociones de los directivos sobre sus empleados, donde dicho sea de paso las mujeres son más emocionales que los hombres (Nájera, 2016).

Los líderes deben motivar a suss empleados, ya que se puede lograr a través de ellos: mejores formas de hacer su trabajo, preocuparse por los clientes, enorgullecerse del trabajo, y ser más productivos (Sabir, 2017). Así mismo, un líder de excelencia muestra la capacidad de inspirar a otros, a través de su propio ejemplo y tomando terstimoniales que le permitan hacer cambios significativos en sus seguidores, tanto en el ámbito profesional 
- laboral, como en el personal (Torres-Flórez, 2020). No obstante, como ya se ha mencionado, el dominio del género masculino en el mercado laboral es determinante, ya que la sexuación en nuestra sociedad genera una forma de apreciación específica en cada cultura, definiendo con base a sus creencias la división del trabajo, la manera de ejercer el poder, así como aspectos sobre la moral (Lamas, 2000). Todo ello, perjudica más a la mujer y genera de alguna forma prácticas de discriminación laboral, incluso por la sencilla razón de ser "físicamente" más débil e identificarse dentro de un grupo "vulnerable" en nuestro ente social, además de que por el rol que juegan en la familia son mucho más propensas a trabajar a tiempo parcial que los hombres (Waaijer, Sonneveld, Buitendijk, Van Bochove y Vander, 2016). Esta situación no es exclusiva de los mexicanos, también en muchso países de América Latina y de orden mundial, existen restricciones para que las mujeres trabajen y ocupen cargos del nivel dirección. Así lo muestra el informe de Grant (2014), donde se menciona que en México únicamente el 28 porciento del personal femenino que trabaja, se encuentra al frente de una jefatura, mientras que en Brasil y Japón, las cifras rondan el 22 y 7 porciento, respectivamente, por mencionar solo esos países.

El sector servicios, al que pertenecen las universidades privadas, ya se ha hecho normal que personal femenino ocupe puestos adminsitrativos como: Secretaria, Apoyo Administrativo, Recepcionista, Intendente, entre otros de nivel operativo. En contraparte, los hombres en la mayoría de los casos se encuentran colocados en los mandos medios y estratégicos. También hay que decir que poco a poco a través del tiempo se va mejorando la participación de las féminas en puestos directivos, no obstante y en particular en México, aún hay estereotipos, creencias, supuestos y en general aspectos culturales, que limitan el acceso de la mujer para realizar funciones de gran responsabilidad laboral, cuestionando su aspecto físico y mental, su rol de madre y esposa (Fischer y Ursul, 2015), es decir, los cuidados que implica el tener hijos y parte de la familia, restringe el poder trabajar más horas.

\section{Materiales y métodos}

El estudio es cuantitativo, descriptivo y transaccional.

El universo poblacional se compone de veinte universidades privadas, ubicadas en los municipios de Pachuca y Muneral de la Reforma, Hidalgo, donde se tienen datos que laboran alrededor de 112 mujeres que ocupan cargos del nivel dirección (Sistema Empresarial Mexicano, 2018).

Considerando aspectos como ubicación, facilidad de acceso, tiempo disponible y entusiasmo de participación, se decidió utilizar un muestro por conveniencia de cincuenta directivas, las cuales manifestaron la necesidad de que se les respetara el anonimato, tanto personal como institucional. Asi mismo y de forma complementaria, se tomó en cuenta que las integran- 
tes de la muestra, necesariamente tuvieran el nombramiento de un puesto directivo, trabajadores bajo su mando, reconocimiento formal del puesto y amplio poder para tomar decisiones dentro de la universidad.

La recolección de datos se llevó a cabo a través de la aplicación de un cuestionario, el cual fue adaptado tomando como base el desarrollado por los autores Mocayo y Zuluanga (2014), para lo cual fue necesario realizar algunas modificaciones, con la finalidad de poder analizar aspectos relacionados con el cargo de dirección que ostentan las féminas lideresas. Los rubros se integran de la siguiente manera:

- Alicientes de acceso al puesto,

- Problemas relacionados con el acceso al puesto,

- Autopercepción de las actividades o funciones realizadas,

- Administración del tiempo, y

- Autopercepción relacionada con sus compañeros hombres.

La confiabilidad del instruemnto cuantitativo fue determinada mediante el cálculo del Coeficiente de Aplha de Cronbach, a través del paquete estadístico SPSS ver 20 en español, el cual fue de 0.92 .

\section{Resultados}

En la tabla 1, se puede observar los datos referentes a la antigüedad laboral que tienen las directivas que participaron en la investigación, encontrando que de las ciencuenta integrantes de la muestra, diez no han cumplido los primeros 5 años, mientras que treinta tienen de 5 a 10 años laborando en su universidad y diez más son las que ya han pasado más de 10 años de su ingreso. Esto no quiere decir que en el cargo laboral como directivas tengan esos años, es decir, desde que tuvieron la oportunidad de trabajar ocupando otros puesto de mayor rango antes de subir como directiva. En promedio la antigüedad es de 7.4 años de todas las mujeres encuestadas.

Tabla 1 Antigüedad laboral

Antigüedad Laboral (años) Total de mujeres

$$
\begin{gathered}
\text { < de } 5 \\
>=5 \text { a } 10 \\
>10 \\
\text { Total }
\end{gathered}
$$

50

Fuente: Elaboración propia con base a los resultados obtenidos

En la sección de preguntas abiertas se les preguntó a las directivas de las universidades, cuál es la proporción que guarda la ocupación de puestos de dirección entre hombres y mujeres, determinando una respuesta promedio del ochenta porciento.es decir por cada 10 cargos 8 son para varones y 2 para damas.

En funsión de los rubros en que se divide el cuestionario aplicado, a continuación se presentan los resultados más relevantes obtenidos, resaltando el entusiasmo con la que se expresaron las mujeres integrantes de la 
muestra, complementando sus respuestas con comentarios y experiencias obtenidas de su propia voz.

En la tabla 2, se aprecia que la mayoría de las directivas (60\%) opina que su principal aliciente en el trabajo es la posibilidad de poder mejorar el sueldo, ya que sienten que es una de las mejores formas de recompensar el esfuerzo y dedicación, todo ello, para obtener un mejor bienestar e ir construyendo con el paso del tiempo un patrimonio sólido. En segundo lugar (31\%) coincidieron en comentar que un buen impulso motivacional es justamente el sentirse satisfachas de su desarrollo y crecimiento profesional, así como de adquirir cada vez más experiencia laboral. Un menor número (9\%) se inclinó por la ayuda al núcleo familiar, toda vez que actualmente la economía de las familias no es proporcional a la inflación en muchos productos y servicios necesarios, por lo que es común que la mayoría de las parejas trabaje e incluso hasta los hijos cuando son mayores de edad.

Tabla 2 Primer rubro y elementos de la encuesta

\begin{tabular}{c|c|c} 
Rubro & Elemento & $\begin{array}{c}\text { Resultado } \\
\mathbf{( \% )}\end{array}$ \\
\hline Alicientes de & $\begin{array}{c}\text { Mejora de } \\
\text { sueldo }\end{array}$ & 60 \\
\cline { 2 - 3 } acceso al puesto & $\begin{array}{c}\text { Satisfacción y } \\
\text { experiencia }\end{array}$ & 31 \\
\cline { 2 - 3 } & $\begin{array}{c}\text { Ayuda al núcleo } \\
\text { familiar }\end{array}$ & 9 \\
\hline
\end{tabular}

Fuente: Elaboración propia con base a los resultados obtenidos

En la tabla 3, se puede observar el segundo rubro del cuestionario aplicado con sus elementos que lo conforman, donde la mayoróa de las féminas encuestadas (45\%) señaló que los problemas y desigualdades que con base a su experiencia han tenido es que muchas veces sus colegas no ven con buenos ojos que una mujer este a su nivel y colocan barreras de diversos tipos para evitar subir de categoría o puesto o bien para desprestigar sus actividades y evidenciar falta de preparación y actitud en el cargo que ocupan. Otro elemento importante que ellas señalan (20\%) es el hecho de ser mamá y tener vástagos menores de edad, lo que implica para los varones señalar o pensar que al tener que cumplir con sus obligaciones como madre y esposa, les impide laborar largas jornadas y disponer de más tiempo, además de los permisos o incapacidades médicas a las que pueden estar sujetas para tener o cuidar de los hijos. Más de la tercera parte (30\%) coincidió en señalar que son comunes las preferencias por contar con conocidos importantes, como el dueño de la empresa, familiares (socios) o los principales directivos, lo cual permite aún la designación directa de nombramientos en distintos cargos y sobretodo las práticas recurrentes de acudir fuera de la jornada laboral a lugares de esparcimiento con los dueños o superiores por parte de los hombres, ya que las mujeres normalmente cuando cumple con su horario de tgrabajo sale apurada a continuar ahora con labores de hogar y familia, hecho que las coloca en desventaja y que reduce las oportunidades de competir "sanamente" por un puesto. 
Afortunadamente, la menor parte de las entrevistadas (5\%), ha sufrido de cuestiones relacionadas con el acoso laboral y sexual, negándose rotundamente a caer en esas prácticas indeseables, no obstante reconocen que hay quienes ceden a este tipo de pretenciones y logran obtener el ascenso más rápido a cargos importantes o bien gozar de ciertos beneficios.

Tabla 3 Segundo rubroy elementos de la encuesta

\begin{tabular}{|c|c|c|}
\hline Rubro & Elemento & $\begin{array}{c}\text { Resultado } \\
(\%)\end{array}$ \\
\hline \multirow{4}{*}{$\begin{array}{l}\text { Problemas rela- } \\
\text { cionados con el } \\
\text { acceso al puesto }\end{array}$} & $\begin{array}{l}\text { Competencia } \\
\text { interna }\end{array}$ & 45 \\
\hline & $\begin{array}{l}\text { Madres con vás- } \\
\text { tagos menores } \\
\text { de edad }\end{array}$ & 20 \\
\hline & $\begin{array}{l}\text { Preferencias por } \\
\text { tener conocidos } \\
\text { importantes }\end{array}$ & 30 \\
\hline & $\begin{array}{c}\text { Negarse al ser } \\
\text { acosada }\end{array}$ & 5 \\
\hline
\end{tabular}

Fuente: Elaboración propia con base a los resultados obtenidos

La tabla 4 refiere al tercer rubro del cuestionario, donde la gran parte de las mujeres participantes del estudio (85\%) declararon tener un nivel de satisfacción en el trabajo muy bueno, ya que su relación vertical y horizaontal es buena, así como con los dueños de las universidades privadas donde laboran. En contraparte, un menor número (15\%) argumenta haber tenido la experiencia de sufrir cierto tipo de actitudes misóginas por parte de sus compañeros o equipos antagónicos, así como envidias y recelos por el puesto directivo que ocupan, es decir, de cada ciencuenta mujeres que ocupan cargos de dirección, 8 sufren de este tipo de situaciones.

Tabla 4 Tercer rubro y elementos de la encuesta

\begin{tabular}{c|c|c} 
Rubro & Elemento & $\begin{array}{c}\text { Resultado } \\
\mathbf{( \% )}\end{array}$ \\
\hline $\begin{array}{c}\text { Autopercepción } \\
\text { de las activida- } \\
\text { des o funciones } \\
\text { realizadas }\end{array}$ & $\begin{array}{c}\text { Nivel de satisfac- } \\
\text { ción al interior } \\
\text { de la empresa }\end{array}$ & $\begin{array}{c}\text { Nivel de insa- } \\
\text { tisfacción al } \\
\text { interior de la } \\
\text { empresa }\end{array}$ \\
\hline
\end{tabular}

Fuente: Elaboración propia con base a los resultados obtenidos

La tabla 5, muestra porcentajes (49, 33 y 18\%) asociados a la opinión de las encuestadas, sobre que tanto les ha mermado el tiempo restante que les queda, después de su jornada laboral, en llevar a cabo acciones de tipo particular o personal, familiar y relacionadas a los menesteres de su casa, respectivamente. En este sentido, para cualquier trabajador, sea hombre o mujer, la jornada diaria de trabajo tiene un efecto en el tiempo que se le puede dedicar a otro tipo de actividades individuales o grupales, no obstante, el personal femenino, por el rol que juega dentro de la sociedad, es la que más responsabilidad tiene en tareas de atención a su familia, dejando en último lugar sus necesidades de tipo personal.

El último rubro referente a la autopercepción relacionada con sus compañeros hombres, en principio como se observa en el gráfico 1, existen desigualdades en relación con el sueldo que reciben mujeres quen ocupan cargos similares a los varones, al me- 
nos 12 de cada 50 entrevistadas, así lo perciben.

Tabla 5. Cuarto rubro y elementos de la encuesta

\begin{tabular}{c|c|c} 
Rubro & Elemento & $\begin{array}{c}\text { Resultado } \\
\text { (\%) }\end{array}$ \\
\hline \multirow{2}{*}{$\begin{array}{c}\text { Administración } \\
\text { del tiempo }\end{array}$} & $\begin{array}{c}\text { Aspectos } \\
\text { personales }\end{array}$ & 49 \\
\cline { 2 - 3 } & $\begin{array}{c}\text { Aspectos } \\
\text { familiares }\end{array}$ & 33 \\
\cline { 2 - 3 } & $\begin{array}{c}\text { Aspectos } \\
\text { hogareños }\end{array}$ & 18 \\
\hline
\end{tabular}

Fuente: Elaboración propia con base a los resultados obtenidos

Gráfico 1. Diferencia salarial mujeres vs hombres

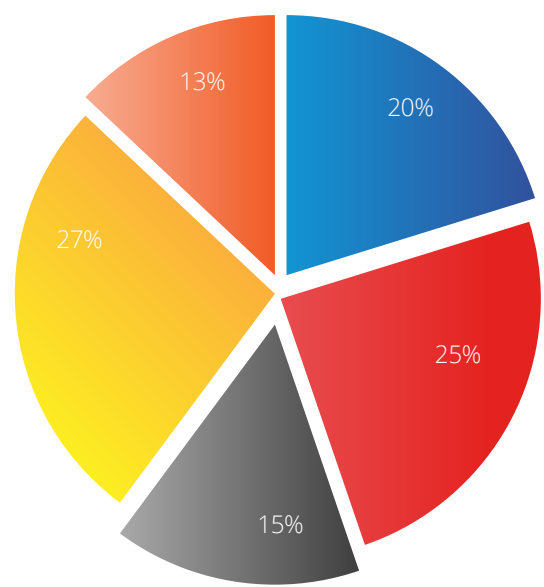

Siempre Casi siempre

Regularmente Casi nunca Nunca

Fuente: Elaboración propia con base a los resultados obtenidos

\section{Discusión}

Los resultados exhiben una realidad muy elocuente, ya que en las universidades privadas objeto de estudio, se sostiene una tradición cultural asociada con la masculinidad hegemóni- ca, que muestra el mandato y control que siguen teniendo los hombres en la mayoría de los puestos directivos, alejando a la mujer de un terreno más equitativo donde pudieran existir oportunidades y mayor igualdad para ellas.

La percepción basada en las experiencias que han tenido las directivas que componen el estudio realizado, muestra por un lado que, al igual que los caballeros, sus principales alicientes que las impulsan y motivan son el recibir un sueldo más justo o competitivo, sentirse satisfechas y reconocidas por sus capacidades laborales, así como lograr aportar recursos económicos para salir adelante con su familia. Esto coincide con lo que expresa León (2003:35) "no todas las relaciones humanas están exclusivamente gobernadas por el tiempo-dinero, pues necesitamos dormir, comer y necesitamos establecer relaciones sociales y afectivas".

Las desigualdades que se hacen patentes de forma no muy recurrente para las directivas de las universidades privadas, tienen relación con rivalidades o celos profesionales de grupos antagónicos que tratan de obtener los mejores cargos y beneficios, sobre todo aquellas que tienen hijos menores de edad y responsabilidades como esposas y amas de casa. Es por ello que los dueños de las empresas deden ser más concientes de estos aspectos emocionales en el trabajo, ya que con el tiempo pueden crear consecuencias negativas en la calidad de vida del personal (Girbés y Martín, 2015).

Finalmente, es indudable que la mujer posee una carga familiar más impor- 
tante que el hombre, ya que además de cuidar a los hijos, es hija y esposa, lo cual se refleja de alguna manera en el ámbito laboral, donde el poco tiempo libre que les queda, lo ocupan para literalmente "correr" a su casa y cumplir con sus demás compromisos del hogar, destinando muy poco tiempo para sus deseos personales.

\section{Conclusiones}

La problemática planteada, merece que se haga un análisis más profundo sobre las principales razones que hoy en día siguen prevaleciendo en la cntralización del poder por parte del género masculino, así mismo, que tanto la percepción ha cambiado en relación a las teorías asociadas con la masculinidad hegemónica, en función de la designación equitativa de puestos directivos en las universidades privadas. En este sentido y a pesar de que se ha ido ganando terreno en la ocupación de algunos cargos importantes, hay investigaciones que aseveran que nunca la sociedad empresarial aceptaría totalmente a las mujeres en cargos ejecutivos (Zabludovsky, 2017).

Es importante divulgar las experiencias que aportan las mujeres que ocupan puestos de buen nivel, ya que poco a poco los directivos y dueños de las empresas privadas, no solo en el ámbito educativo privado, sino en cualquier sector, deberán sensibilizarse con aspectos diferenciadores o propios del rol que juega la mujer dentro de nuestra sociedad. Es decir, el hecho de no poder a veces quedarse tiempo extra o acudir a eventos so- ciales fuera de la empresa, donde se acostumbra a tomar la copa y culminar a altas horas de la noche, o donde el salario de cargos o puestos iguales son distintos entre un hombre y mujer, entre otros aspectos, no significa perder la oportunidad de competir honestamente por un cargo o puesto importante (BBC Mundo, 2016).

\section{Referencias}

BBC Mundo (2016). Los verdaderos motivos detrás de la diferencia salarial entre hombres y mujeres. Recuperado de https://www.bbc.com/mundo/noticias-37217241

Biedma, J. (2017). La mujer directiva. La presencia de la mujer en los consejos de administración de las compañías del Ibex 35. Dossiers Feministes, 22(1), 13-27. doi: http://dx.doi.org/10.6035/ Dossiers.2017.22.2

Blanco Rangel, I. (2016). Apuntes sobre Colombia a propósito de su internacionalización y otros desafíos. Aglala, 7(1), 209-224. Recuperado de http:// revistas.curnvirtual.edu.co/index.php/ aglala/article/view/905

Bonnafé, J. (2016). Principios para el empoderamiento de las mujeres en las empresas. Recuperado de http://www.onu.org.mx/wp-content/ uploads/2016/12/Empoderamiento-mujeres-en-empresas.pdf

Buquet, A. y Moreno, H. (2017). Trayectorias de mujeres. Educación técnico-profesional y trabajo en México. Recuperado de https://www.researchgate.net/publication/318684416_Trayectorias_de_mujeres_Educacion_tecnico-profesional_y_trabajo_en_Mexico

Castro Alfaro, D., \& Castro Alfaro, A. (2016). La formación profesional de 
los ingenieros de la Universidad Inca Garcilaso de la Vega desde el punto de vista de los egresados. Conocimiento Global, 1(1), 1-13. Recuperado a partir de http://conocimientoglobal. org/revista/index.php/cglobal/article/ view/20

Conacanaco Servytur México (2017). Indicadores Hidalgo. Recuperado de https://www.concanaco.com.mx/documentos/indicadores-estados/Hidalgo. pdf

Díez, E. (2015). Códigos de masculinidad hegemónica en educación. Revista lberoamericana de Educación, 68(1), 7998. Recuperado de https://rieoei.org/ historico/documentos/rie68a04.pdf

Fischer, L. y Ursul, J. (2015). Percepción del rol que desempeñan las mujeres en puestos directivos. Recuperado de http://congreso.investiga.fca.unam. $\mathrm{mx} /$ docs/xx/docs/11.11.pdf

Girbés, B. y Martín, R. (2015). Celos y envidia en el trabajo: una revisión de los últimos 20 años. Apuntes de Psicología, 33(3), 127-136. Recuperado de http://www.apuntesdepsicologia.es/ index.php/revista/article/view/569

Grant, T. (2014). Women in Business Report 2014. Recuperado de http://www. grantthornton.es/publicaciones/estudios/Grant-Thornton-estudio-Mujeres-directivas-2014.pdf

Horbath, J. y Gracia, A. (2014). Discriminación laboral y vulnerabilidad de las mujeres frente a la crisis mundial en México. Economía, Sociedad y Desarrollo, 14(45), 465-495. Recuperado de http://www.scielo.org.mx/pdf/est/ v14n45/v14n45a6.pdf

Independiente (2020). Hidalgo, con más mujeres en puestos directivos. Recuperado de https://www.elinde- pendientedehidalgo.com.mx/hidalgo-con-mas-mujeres-en-puestos-directivos/

Kemal, S. (2015). Understanding Leadership and Factors that Influence Leaders' Effectiveness. European Journal of Business and Management, 7(33),154-167. Recuperado de https://www.researchgate.net/publication/313636384_Understanding Leadership_and_Factors_that_Influence_Leaders\%27_Effectiveness

Lamas, M. (2000). Diferencias de sexo, género y diferencia sexual. Cuicuilco, 7(18), 1-24. Recuperado de https://www.redalyc.org/pdf/351/35101807.pdf

Labarcés Ballestas, C. (2015). Aspectos de innovación schumpeteriano. Dictamen Libre, (17), 73-80. https://doi. org/10.18041/2619-4244/dl.17.3082

León, M. (2003). Mujeres y trabajo: cambiosimpostergables. Recuperado de https://www.alainet.org/publica/mujtra/mujeres-trabajo.pdf

Moncayo, B. y Zuluanga, D. (2014). Estrategias y obstáculos en el acceso femenino a puestos directivos en la academia. Recuperado de http://premio. investiga.fca.unam.mx/docs/ponencias/2014/7.1.pdf

Nájera, S. (2016). Liderazgo e inteligencia emocional. Revista Innova, 1(1), 19-24. Recuperado de http://revistas.uide. edu.ec/index.php/innova/index

O'riordan, J. (2017). The practice of human resource management. Recuperado de https://www.ipa.ie/_fileUpload/Documents/THE_PRACTICE_OF_HRM.pdf

Ocañas, E. (2019). Un análisis de la brecha salarial por género: caso aplicado a una empresa de manufactura en Escobedo, México. Revista GEON, 
6(2), 82-89. Recuperado de http://revistageon.unillanos.edu.co/index.php/ geon/article/view/173/159

Sabir. A. (2017). Motivation: Outstanding Way to Promote Productivity in Employees. American Journal of Management Science and Engineering, 2(3), 3540. doi: http://dx.doi.org/10.11648/j. ajmse.20170203.11

Sistema Empresarial Mexicano (2018). SIEM. Recuperado de https://siem. gob.mx/

Torres-Flórez, D. (2020). La generación de valor entre las personas y las organizaciones. Revista GEON, 7(1),4-8. https://doi.org/10.22579/23463910.211

Universia México (2018). ¿Qué requisitos piden las empresas para un puesto de directivo? Recuperado de https://noti- cias.universia.net. $\mathrm{mx} /$ practicas-empleo/noticia/2018/02/06/1157864/ requisitos-piden-empresas-puesto-directivo.html

Waaijer, F., Sonneveld, H., Buitendijk E., Van Bochove C., Vander Weijden, C. (2016). The Role of Gender in the Employment, Career Perception and Research Performance of Recent PhD Graduates from Dutch Universities. PLOS ONE, 11(10), 1-16. Recuperado de https://journals.plos.org/plosone/ article/file?id=10.1371/journal.pone.0164784\&type=printable

Zabludovsky, G. (2017). Las mujeres en México: trabajo, educación superior y esferas de poder. Revista Política y Cultura, 1(28), 9-41. Recuperado de http://www.scielo.org.mx/pdf/polcul/ n28/n28a2.pdf 